\title{
RESEARCH
}

Open Access

\section{Role of abbreviated MRI protocol for screening of HCC in HCV related cirrhotic patients prior to direct-acting antiviral treatment}

Nora Nabil Abdou Ahmed*, Sahar Mohamed El Gaafary, Remon Zaher Elia and Essam Mohamed Abdulhafiz

\begin{abstract}
Background: Chronic HCV infection is a global health problem causing progressive hepatic fibrosis, cirrhosis, and ultimately hepatocellular carcinoma (HCC). Recent advantage of direct-acting antiviral drugs (DAA) with a high sustained virologic response (SVR) reduces overall HCV-related morbidity and mortality, yet recent studies report a high recurrence rate of HCC after DAA; this calls availability of a reliable screening method to properly exclude HCC before DAA treatment.

The primary objective of our cohort study was to assess the feasibility of an abbreviated MRI protocol as a screening tool for the detection of hepatic focal lesions/early HCC in patients with HCV-related liver cirrhosis. The study included 41 patients with HCV-related cirrhosis candidates to DAA therapy. All patients underwent routine screening for HCC by combined abdominal ultrasound and serum alfa-fetoprotein. An abbreviated MRI protocol (Abr-MR) including combined T2-weighted image and diffusion-weighted imaging (DWI) followed by dynamic contrast-enhanced MRI (CE-MRI) was performed for all subjects, assessing for presence and characterization of focal lesions.

Results: For all included 41 patients, no elevation of the alpha-fetoprotein was shown. Ultrasound detected a single focal lesion in one patient. Abbreviated MR demonstrated 15 focal lesions; for detected lesions, 1 lesion shows "shine though" and the rest showing moderate and high degrees of restriction. CE-MRI characterized lesions as 1 lesion = LIRADS-1, 3 lesions = LR-M, and 11 lesions = LR-5.The standard screening using combined ultrasound and alpha-fetoprotein had sensitivity, specificity, PPV, and NPV of $6.6 \%(95 \% \mathrm{Cl}=0.0034-0.29), 100 \%(95 \% \mathrm{Cl}=0.89-$ 1.000), $100 \%(95 \% \mathrm{Cl}=0.051-1.000)$, and $68.8 \%$ (95\% Cl=0.54-0.80). Abr-MR protocol showed sensitivity, specificity, PPV, and NPV of 100\% for all parameters (95\% Cl $=0.79-1.000,0.89-1.000,0.79-1.000$, and 0.89-1.000 respectively).

Conclusion: In our study, we demonstrate the superiority of a proposed cost-effective Abr-MR protocol in the detection of hepatic focal lesions and small-sized HCC compared to routine screening using alpha-fetoprotein and ultrasound in HCV-related liver cirrhosis.
\end{abstract}

Keywords: Ultrasound, Diffusion-weighted MRI, Hepatocellular carcinoma, Direct-acting antiviral

*Correspondence: noranabil15@yahoo.com

Radiodiagnosis Department, Faculty of Medicine, Ain Shams University, Cairo,

Egypt

(c) The Author(s). 2020 Open Access This article is licensed under a Creative Commons Attribution 4.0 International License, which permits use, sharing, adaptation, distribution and reproduction in any medium or format, as long as you give appropriate credit to the original author(s) and the source, provide a link to the Creative Commons licence, and indicate if changes were made. The images or other third party material in this article are included in the article's Creative Commons licence, unless indicated otherwise in a credit line to the material. If material is not included in the article's Creative Commons licence and your intended use is not permitted by statutory regulation or exceeds the permitted use, you will need to obtain permission directly from the copyright holder. To view a copy of this licence, visit http://creativecommons.org/licenses/by/4.0/. 


\section{Background}

Chronic HCV infection is a global health problem that affects millions of people worldwide. HCV is associated with several hepatic and extra hepatic disorders, including several malignancies like colon cancer and cancer esophagus .The burden of HCV-related disorders is influenced by the number of new and existing cases and the natural history of the infection. The natural history of $\mathrm{HCV}$ is affected by several demographic, virological, clinical, and lifestyle factors [1]. In patients with chronic HCV infection, progressive hepatic fibrosis leading to cirrhosis (in $15-35 \%$ of patients after $25-30$ years duration) is responsible for most of HCV-related morbidity and mortality, including hepatocellular carcinoma (HCC) [1]. In several cohort studies, the risk of HCC in HCV-infected people was tenfold higher compared to $\mathrm{HCV}$-negative individuals, and risk estimates of 20-25fold have been reported in case-control studies. Once $\mathrm{HCV}$-related cirrhosis is established, HCC develops at an annual rate of up to $8 \%$ (average $2-3 \%$ ), thus making cirrhosis an important precancerous condition. The interval from infection to $\mathrm{HCC}$ has been estimated to be 30 years, although latent periods of $40-50$ years have been observed [2].

International guidelines for screening of $\mathrm{HCC}$ in cirrhotic patients suggest an ultrasound every 6 months as the first level of investigation. The main goal is diagnosing early $\mathrm{HCC} /$ dysplastic nodule; however, such a goal remains difficult due to the low sensitivity of such protocol $(60 \%)$ [3].

$\mathrm{HCC}$ is unique in having imaging characteristics on contrast-enhanced computed tomography (CE-CT) or contrast-enhancing magnetic resonance imaging (CEMRI) allowing for its accurate diagnosis. This primarily depends on the enhancement criteria of the suspected lesion relative to the background liver in the hepatic arterial, portal venous, and subsequent phases [4].

Hence, CE-CT/CE-MRI is most widely used for characterizing $\mathrm{HCC}$ in patients with cirrhosis. This is due to the increased ability of arterial phase enhancement to visualize early, small lesions. Although the sensitivity of helical CT for depicting hepatocellular carcinoma in patients with cirrhosis has been well documented to be from 50 to $70 \%$, CE-MRI has shown higher figures of sensitivity and specificity for HCC detection and characterization especially for early/small-sized HCC [5].

However, MRI examinations have limited accessibility, high cost, long scan time, and the risk of contrast-related side effects. When performed without the use of contrast agents, the risk of contrast-related adverse events is eliminated, and both the cost and examination time can be reduced. With this in mind, non-contrast MRI is potentially a good alternative surveillance tool for HCC [6].
Considering non-enhancing MRI adding diffusionweighted imaging (DWI) improves detection and-to an extent-characterization of HCC. Hence, DWI carries a potential capacity for improving patient care through its capacity of insight into tumor biology [7].

\section{Methods \\ patients}

A prospective study done on forty-one patients with liver cirrhosis recruited from the HCV clinic at the hospital institution. The procedure was explained in full, and the informed consent was signed by them. The study was conducted during the period from May 2018 to April 2019.

\section{Inclusion criteria}

- HCV positive patients with liver cirrhosis are candidates for antiviral therapy

\section{Exclusion criteria}

- Other causes of liver cirrhosis (HBV, NASH, alcoholic liver cirrhosis, immune-related, primary biliary cholangitis, sclerosing cholangitis, and idiopathic causes of liver cirrhosis)

- Contraindication for MRI (electronic pacemakers, MRI incompatible implanted devices, intolerant patients)

- Previous locoregional therapy for focal lesion

- Contraindication to MR contrast administration(GFR $<30 \mathrm{ml} / \mathrm{min}$ )

\section{Technique}

All patients are subjected to clinical and laboratory assessment, including viral markers, liver function tests, HCV-PCR, and serum alpha-fetoprotein assay.

A liver ultrasound was performed by using (GE healthcare) machine with curvilinear probe $(3-5 \mathrm{MHz})$ and applied gel on patient lying supine on the examination table.

After completion of the sonographic assessment, MRI examinations were performed including the Abr-MRI and CE-MRI protocols. The MR performed with $1.5 \mathrm{~T}$ machine (Philips Achieva; Philipsmedical systems, Eindhoven, Netherland), using 16-channel phased-array torso coil (Sense XL Torso; Philips Healthcare) covering the length of the abdomen.

Patients were positioned supine-head first-on the MRI table, then the abbreviated MRI (Abr-MR) was performed including T2 weighted fast spin-echo (T2-FSE) and T2 weighted imaged spectral resaturation with inversion recovery (T2- SPIR), and diffusion-weighted image (DWI) map was performed for using $3 b$ values 0 , 200, and 800 (with TR $1300 \mathrm{~ms} \&$ TE $64 \mathrm{~ms}$ ), with 
corresponding ADC mapping. CE-MRI was performed afterward using gadopentetate dimeglumine (Omnivist ${ }^{\circ}$ ) injected through an antecubital intravenous catheter at a rate of $3 \mathrm{ml} / \mathrm{min}$ over $15 \mathrm{~s}$ and a dose of $0.2 \mathrm{ml} / \mathrm{kg}$ followed by saline chaser of $30-40 \mathrm{ml}$ at a rate of $1-2 \mathrm{~mL} /$ s. Dynamic contrast-enhanced sequences were acquired using T1 weighted high-resolution isotropic volume examination (THRIVE) acquired before (pre-contrast) and after contrast injection at $1520 \mathrm{~s}$ (arterial phase), $40 \mathrm{~s}$ (portal phase), $60 \mathrm{~s}$ (venous phase), and $180 \mathrm{~s}$ (delayed phase). All contrast sequences were acquired at the axial plane.

\section{Statistical data analysis}

Data were coded and entered using the statistical package SPSS version 25. It was summarized using descriptive statistics: frequencies were stated as numbers of observations and percentages for categorical variables. Means and standard deviations were used as measures of central tendency for parametric numerical variables.

Inferential statistical analyses for assessment of the relation between categorical variables were performed using both contingency tables with chi-squared tests for observed and expected values and testing for correlation using the spearman-rho test. All tests are considered statistically significant at a $p$ value equal to or less than 0.05 .

Diagnostic performance indices including sensitivity, specificity, positive and negative predictive values, likelihood ratios, and overall accuracy were calculated using contingency tables.

Data was presented by graphs, bar charts, and pie charts as well as tables.

\section{Results}

Our study was carried on a total of 41 patients (22 males, 19 females) with a mean age of $53.4 \pm 9.17$ years (males $=50.74 \pm 8.5$, females $=57.31 \pm 8.8$ years $)$.

For the Abr-MRI, a total of 15 focal lesions were detected on either T2WI/T2-SPIR or DWI in 10 patients $($ male $=12$, females $=3)$. The lesions were most frequently present in segment VIII $(n=7,46.7 \%)$ followed by segments VI, V, and II $(n=2,13.3 \%)$ and segments VII and III $(n=1,6.7 \%)$. The mean diameter of the focal lesions was ranging from 1.4 to $3.8 \mathrm{~cm}$. Two focal lesions seen at segment II, segment V, and segment VI and their measurements ranging from 1.9 to $2.3 \mathrm{~cm}, 1$ to $4.7 \mathrm{~cm}$, and 1 to $2.2 \mathrm{~cm}$, respectively; seven focal lesions seen at segment VIII ranging from 2 to $4.4 \mathrm{~cm}$; and one focal lesion seen at segment III and segment VII measuring 3.2 $\mathrm{cm}$ and $1.9 \mathrm{~cm}$, respectively (Table 1 ).

T2WI detected only 13 lesions (intermediate to high $=1$, bright $/$ high signal $=12$ ) and detected no lesions in 33 cases. Combining the DWI and ADC, 14 lesions exhibited true restriction $(\mathrm{ADC}=0.72+/-0.08)$, and 1 lesion exhibited $\mathrm{T} 2$ shine through $(\mathrm{ADC}=2.00)$ (Figs. $1 \mathrm{~b}, \mathrm{c}$ and $2 \mathrm{~b}-\mathrm{d}$ ). Utilizing the T2WI/T2-SPIR only for focal lesion detection showed relatively lower figures of sensitivity $(86.67 \%, 95 \%$ $\mathrm{CI}=59.5-98.3 \%)$ and good negative predictive values (93.9\%, 95\% CI = 81.01-98.26), with otherwise excellent specificity and positive predictive values of $100 \%$. Adding the DWI/ADC into the proposed Abr-MR protocol for detection of focal lesions increased sensitivity and NPV for $100 \%$ retaining specificity and positive predictive value at $100 \%$ also $(95 \% \mathrm{CI}=0.79-1.000,0.89-1.000,0.79-1.000$, and $0.89-1.000$, respectively).

CE-MRI detected 15 lesions, which were characterized based on LI-RADS v2018 including 11 LR-5 (definite HCC), 3 LR-M (malignant-not compatible with HCC), and 1 LR-1 (hemangioma); 31 cases were negative for focal lesions (Tables 2 and 3) (Figs. 1b, c and 2b-d).

There was statistically significant difference between lesions classified as high malignant probability (LR-5, LR-M) versus low malignant probability (LR1, no lesion) based on both T2WI/T2-SPIR $\left(\chi^{2}=32.796, p<0.001\right)$ and DWI/ADC characteristics $\left(\chi^{2}=46.00, p<0.001\right)$; moreover, we found strong correlation between detectability (signal brightness) on T2 signal and ( $r=0.838$, $p<0.001)$ stronger correlation between diffusion restriction and probability of malignant focal lesions $(r=0.977$, $p<0.001$ ).

Additionally, the Abr-MR protocol had the same figures of specificity, sensitivity, PPV, and NPV for the detection of lesions of high malignant probability based on the presence of true diffusion restriction. Ultrasound examination in this study detected 1 focal lesion in 1 patient, with the remaining 40 no lesions detected (Figs. 1a and 2a).

Comparing the ultrasound results to the CE-MRI protocol, the ultrasound detected only 1 out of 15 focal lesions and failed to detect 14 . In this study group, ultrasound showed extremely low sensitivity $=6.67 \%$ (95 CI $=0.17-31.95 \%)$ and relatively low NPV $=68.9 \%(95 \% \mathrm{CI}$ $=65.92-71.71 \%)$, while it showed excellent specificity $=$ $100 \%(95 \% \mathrm{CI}=88.78-100 \%)$ and $\mathrm{PPV}=100 \%$.

Table 1 Distribution frequency of the hepatic focal lesions according to gender and liver segment

\begin{tabular}{|c|c|c|c|c|c|c|c|c|c|}
\hline & \multicolumn{2}{|c|}{ Gender } & \multicolumn{7}{|l|}{ Segment } \\
\hline & Male & Female & $\|$ & III & V & $\mathrm{VI}$ & VII & VIII & Total \\
\hline Number of lesions & 12 & 3 & 2 & 1 & 2 & 2 & 1 & 7 & 15 \\
\hline Size of lesions & & & 1.9 to $2.3 \mathrm{~cm}$ & $3.2 \mathrm{~cm}$ & 1 to $4.7 \mathrm{~cm}$ & 1 to $2.2 \mathrm{~cm}$ & $1.9 \mathrm{~cm}$ & 2 to $4.4 \mathrm{~cm}$ & 1.4 to $3.8 \mathrm{~cm}$ \\
\hline
\end{tabular}


(A)
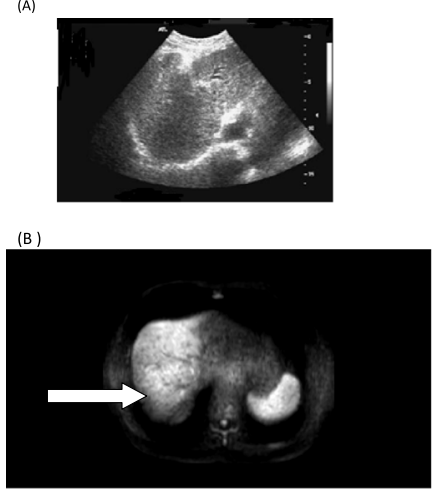

(DWI)

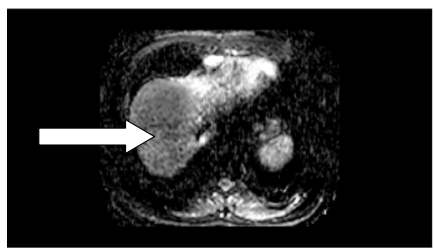

$A D C$ value $=0.5$

(c)

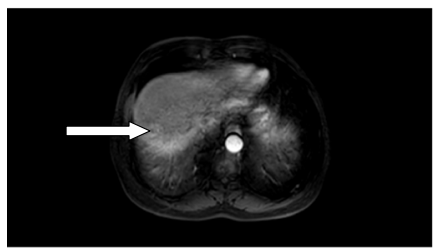

(1)

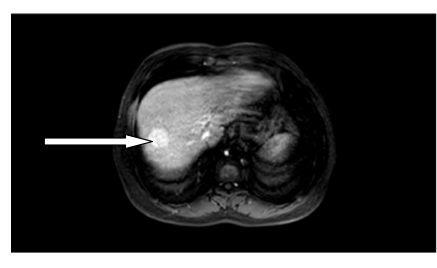

(2)

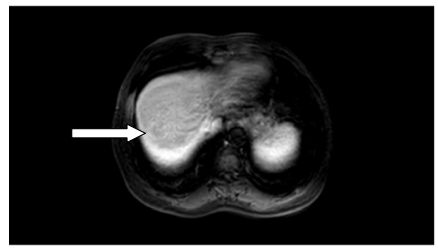

(3)

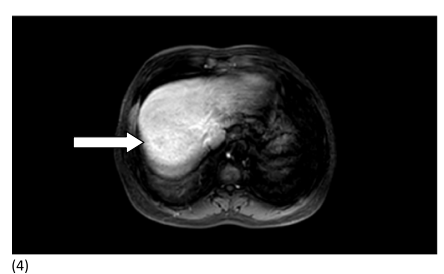

Fig. 1 A 56-year-old male patient underwent an ultrasound and abbreviated MRI protocol followed by standard contrast-enhanced dynamic MRI. a Ultrasound of the liver showed a coarse echo pattern with no visualized focal lesion. b One hepatic focal lesion is noted with true restricted diffusion at segment VIII with ADC value of 0.5; the patient underwent conventional contrast-enhanced MRI for further characterization of this focal lesion. c On contrast study, the lesion shows enhancement pattern consistent with HCC early arterial enhancement with washout on porto-venous with pseudocapsular enhancement

\section{Discussion}

Hepatocellular carcinoma (HCC) is considered the major cause of mortality in patients with hepatitis $\mathrm{C}$ virus (HCV)-related liver cirrhosis; hence, screening for HCC is mandatory for all cirrhotic patients. The advantage of direct-acting antiviral (DAA) therapy for $\mathrm{HCV}$ achieved sustained virologic responses after 12 weeks of therapy (SVR12) of 90-93\%, however, several studies demonstrated conflicting results for reduction of the risk of HCC in patients treated by DAA where some studies showed no significant reduction of the risk of development of HCC following DAA treatment, whereas other studies claimed a relatively higher incidence of $\mathrm{HCC}$ following treatment, especially in patients with treated HCC (recurrence). Moreover, fewer studies showed that the HCC pattern in patients receiving DAA was more aggressive with relatively poor prognosis compared to patients not receiving DAA [8]. All the previous data calls for the presence of a reliable screening method to properly exclude the presence of hepatic focal lesions with the potential for being an HCC before the onset of treatment. In this study, the sensitivity of US for detection of early or small HCC was low measuring $6.6 \%$. Multiple studies were also performed to document the efficacy of ultrasound and its combination with AFP for early detection of HCC; recent different meta-analysis studies are carried by Tzartzeva et al 2018, Singal et al 2012, and Kim 2016 et al who documented combined sensitivity of US with AFP for early stage to be $63 \%$, $90 \%$, and $32.6 \%$ which also decreased down to $47 \%, 44 \%$, and $25 \%$ when the US used alone as a screening tool [810]. The low sensitivity of the US as a screening tool in this study was attributed to using US modality as the sole screening tool rather than the combined US with AFP as well as involvement of patient with only cirrhotic liver where the US shows heterogeneous hepatic texture reducing its sensitivity to discriminate small focal lesion. Albeit, the low sensitivity and negative predictive values documented by the various studies fail to reliably detect early/small HCC or dysplastic nodules despite the convenience, wide availability, and low cost of ultrasound examination for screening. It is of special importance in patients undergoing treatment by direct-acting antiviral (DAA) agents since several recent studies have established 


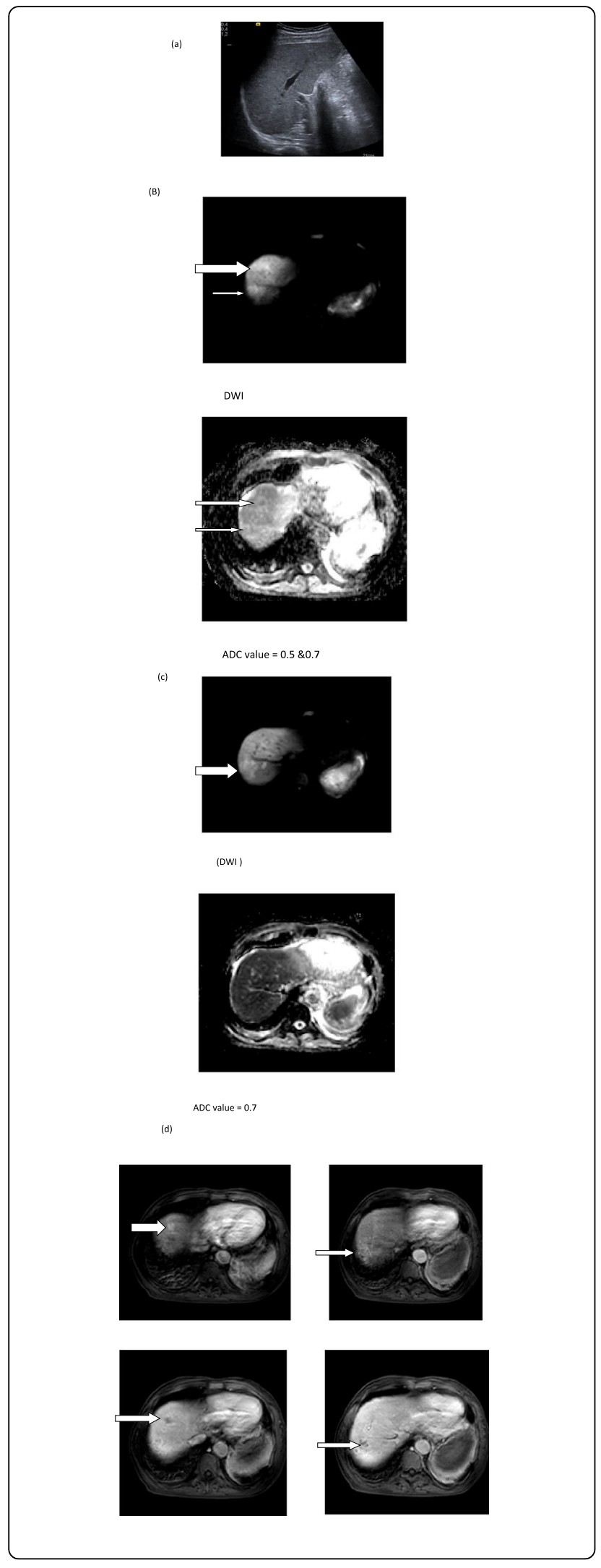

Fig. 2 A 66-year-old male patient underwent an ultrasound and abbreviated MRI followed by standard contrast-enhanced dynamic MRI. a US shows coarse liver echo pattern with no focal lesions. b, c Three hepatic focal lesions are noted (two at segment VIII and one at segment VI) with true restricted diffusion, with $\mathrm{ADC}$ value $0.5,0.7$, and 0.7 , respectively; the patient underwent conventional contrast-enhanced MRI for further characterization of these focal lesions. $\mathbf{d}$ On contrast study, the lesions show enhancement pattern consistent with HCC early arterial enhancement with washout on porto-venous and delayed phase

the high incidence of tumor recurrence, rapid development or development of aggressive patterns of HCC, and most recent guidelines for DAA therapy recommend against using such therapy in patients with untreated HCC. This, in turn, calls for more reliable methods for the exclusion of HCC especially in its early stages before starting the DAA therapy. A method that has shown promising results for the detection of HCC is MRI-diffusionweighted imaging (DWI). In our study, we aimed to examine for the feasibility of a shortened or abbreviated MRI protocol based on a combination of DWI/ADC map and T2 sequence to provide both reduced time and cost which might enable for such MRI protocol as screening for early HCC in cirrhotic patients selected for DAA therapy. In this study, we showed an impressively high sensitivity and negative predictive values (both 100\%) for detection of HCC (lesions categorized as LIRADS LR-4 or LR-5) based on DWI and ADC characteristics of the lesions, in a total of 15 hepatic focal lesions detected in 10 patients, indicating an extremely high reliability for exclusion of such lesions in such patient population. Moreover, the specificity and positive predictive values shown in our study for such LR-4 and LR-5 lesions were also high $(92.3 \%$ and $91.6 \%$, respectively) indicating not only the excellent ability for the exclusion of such lesions but also very good reliability for characterizing those lesions as early/small HCC. This study results agreed with Nasu et al. 2012 who showed a sensitivity of $91.2 \%$ for detection of small-sized HCC (mean diameter of $2.9 \mathrm{~cm}$ ) based on bright DWI signal and low ADC value. Compared to the nearby liver parenchyma [11], however, the comparatively higher figure of sensitivity in this study may be attributable to the fact that Nasu et al. compared the DWI/ADC to surgical

Table 2 Frequency and percentages of the focal lesions according to the LIRADS/CE-MRI characterization

\begin{tabular}{lll}
\hline $\begin{array}{lll}\text { CE-MRI } \\
\text { criteria/ } \\
\text { LIRADS }\end{array}$ & Focal lesion & \\
\cline { 2 - 3 } Lrequency & Percent \\
\hline LR-5 & 11 & 23.9 \\
Negative & 31 & 67.4 \\
LR-1 & 1 & 2.2 \\
LR-M & 3 & 6.5 \\
\hline
\end{tabular}


Table 3 Sensitivity, specificity, NPV, and PPV of US, abbreviated MRI protocol with and without ADC value in the detection of a malignant focal lesion in correlation with CE-MRI

\begin{tabular}{lllll}
\hline & Sensitivity & Specificity & NPV & PPV \\
\hline Ultrasound & $6.6 \%$ & $100 \%$ & $68 \%$ & $100 \%$ \\
Abbreviated MRI without ADC value & $96.8 \%$ & $93.3 \%$ & $100 \%$ & $100 \%$ \\
Abbreviated MRI with ADC value & $100 \%$ & $100 \%$ & $100 \%$ & $100 \%$
\end{tabular}

specimen and histopathological confirmation of $\mathrm{HCC}$ while in this study, we compared the lesions to the standard multiparametric MRI protocol and LIRADS categories. Moreover, the total number of lesions in this study [12] was less than the number included in Nasu et al. study (125). A retrospective study done by Piana et al. [5] on 91 patients with $109 \mathrm{HCC}$ assessing the sensitivity of DWI for detection of HCC in correlation with histopathology; two blinded readers assess HCC being hyper intense with a sensitivity of $89 / 109$ (82\%) and 79/109 (72\%) and decreased to be sensitivity of $74 \%$ and $64 \%$ in tumors less than $20 \mathrm{~mm}$, and there was an agreement between the two readers. Another cohort study done by Shingaki et al. [13], focusing on small sized tumor (20 $\mathrm{mm}$ or less), found that sensitivity being $78.8 \%$ of HCC of 66 pathologically proven lesions were hyper intense to back ground liver. Another retrospective study by Zhao et al. [14] made on HCC lesions and determined for lesions between 0.6 and $2 \mathrm{~cm}$ with an average size of $1.5 \mathrm{~cm}$ was visible on DWI with a sensitivity of $79 \%$. Also, a retrospective study done by Park et al. [15] to estimate the accuracy of DWI in detecting HCC using explanted liver as a gold slandered, and tumor ranged from 0.3 to $6.2 \mathrm{~cm}$ DWI had a sensitivity of $75.8 \%$ and approaches $90 \%$ in lesions more than $20 \mathrm{~cm}$. Low et al. [12], a study for evaluation of the role of added DWI to conventional dynamic MRI study and use of conventional dynamic study alone in detection of a $\mathrm{HCC}$, found that the sensitivity value for use of combined technique was $97.8 \%$, and those use of conventional study alone without DWI was $85.11 \%$. The positive predictive value for combined technique was $97.8 \%$, and for the conventional technique alone was $92.8 \%$. So in this study, the use of added DWI causes higher sensitivity in detection of HCC than dynamic MRI alone .The major advantage of DWI over the USA shown in our study is the less falsepositive results, which will reduce the expense of screening programs as fewer patients will progress into an expensive diagnostic investigation as conventional dynamic contrast-enhanced MRI.

\section{Conclusion}

In our study, we were able to demonstrate the superiority of a proposed cost-effective abbreviated MRI protocol in the detection of hepatic focal lesions and small-sized HCC compared to the routine screening protocol of combined alpha-fetoprotein and transabdominal ultrasound imaging for HCV-related liver cirrhosis. The drawbacks in this study are that the MRI machine is not easily available in remote places, not cheap as US machine, some peripheral small hepatic focal lesion could be missed owing to motion artifact (respiratory motion), or at left lateral lobe due to cardiac motion; however, in comparison to the US machine, still, the MRI machine can detect the majority of clinically significant cancers and has significantly lower false-positive rate than screening ultrasound.

Also, this study can be used as an alternative for the detection and characterization of HCC, especially in patients with impaired renal function or contrast allergies precluding the use of iodinated (CT) contrast media as the majority of patients with liver cirrhosis had hepatorenal syndrome so the use of abbreviated MRI is to avoid element of nephropathy.

\begin{abstract}
Abbreviations
HCC: Hepatocellular carcinoma; DWI: Diffusion-weighted image; HCV: Hepatitis C virus; DAA: Direct-acting antiviral; ADC: Apparent diffusion coefficient; NPV: Negative predictive value; PPV: Positive predictive value; MRI: Magnetic resonance imaging; SD: Standard deviation; LI-RADS: Liver imaging and data reporting system; Abr-MRI: Abbreviated MRI; NASH: Nonalcoholic steatohepatitis; SVR: Sustained virologic response
\end{abstract}

Acknowledgements

The authors thank all the study participants for their patience and support.

Authors' contributions

NNA: reviewing literature, data collection and analysis, perform statistical analysis, write and revise the manuscript, and prepare figure and tables. SMELG: reviewing literature, data analysis, and manuscript editing. RZE: suggest and develop the research idea, reviewing literature, and data analysis. EMAH: reviewing literature, share in statistical analysis, data analysis, and manuscript editing. All authors read and approved the final manuscript.

Funding

This study had no funding from any resource.

\section{Availability of data and materials}

The datasets used and/or analyzed during the current study are available from the corresponding author on reasonable request.

\section{Ethics approval and consent to participate}

The study was a prospective study. This study was approved by the Research Ethics Committee of the Faculty of Medicine at Ain shams University in Egypt on 10 May 2018: Reference Number of approval: FWA 000017585. All patients included in this study gave written informed consent to participate in this research. If the patient was unconscious at the time of the study, written informed consent for their participation was given by their legal guardian.

\section{Consent for publication}

All patients included in this research gave written informed consent to publish the data contained within this study. If the patient was unconscious 
when consent for publication was requested, written informed consent for the publication of this data was given by their legal guardian.

\section{Competing interests}

The authors declare they have no competing interests.

Received: 24 February 2020 Accepted: 5 May 2020

Published online: 16 June 2020

\section{References}

1. Aaron P. Thrift, Hashem B. El-Serag, Fasiha Kanwal (2017): Global epidemiology and burden of HCV infection and HCV-related disease. Nat Rev Gastroenterol Hepatol; 14:122-132.

2. El-Serag HB (2012) Epidemiology of viral hepatitis and hepatocellular carcinoma. Gastroenterology 142:1264-1273

3. Singal A, Volk ML, Waljee A, Salgia R, Higgins P, Rogers MA (2009) Metaanalysis: surveillance with ultrasound for early-stage hepatocellular carcinoma in patients with cirrhosis. Aliment Pharmacol Ther:37-47

4. Manini MA, Sangiovanni A, Fornari F, Piscaglia F, Biolato M, Fanigliulo L et al (2014) Clinical and economical impact of 2010 AASLD guidelines for the diagnosis of hepatocellular carcinoma. J Hepatol 60:995-1001

5. Piana G, Trinquart L, Meskine N, Barrau V, Beers BV, Vilgrain V (2011) New MR imaging criteria with a diffusion-weighted sequence for the diagnosis of hepatocellular carcinoma in chronic liver diseases. J Hepatol 55:126-132

6. Kim SY, An J, Lim YS, Han S, Lee JY, Byun JH, Won HJ, Lee SJ, Lee HC, Lee YS (2017) MRI With Liver-specific contrast for surveillance of patients with cirrhosis at high risk of hepatocellular carcinoma. JAMA Oncol 3(4):456-463

7. Nchingolo R, De Gaetano AM, Curione D et al (2014) Role of diffusionweighted imaging, apparent diffusion coefficient and correlation with hepatobiliary phase findings in the differentiation of hepatocellular carcinoma from dysplastic nodules in cirrhotic liver. Eur Radiol

8. Tzartzeva K, Obi J, Rich NE, Parikh ND, Marrero JA (2018) Surveillance imaging and alpha fetoprotein for early detection of hepatocellular carcinoma in patients with cirrhosis: a meta-analysis. Gastroenterology 154(6):1706-1718

9. Singal AG, Conjeevaram HS, Volk ML et al (2012) Effectiveness of hepatocellular carcinoma surveillance in patients with cirrhosis. Cancer Epidemiol Biomark Prev 21:793-799

10. Kim SY, An J, Lim YS et al (2017) MRI with liver specific contrast for surveillance of patients with cirrhosis at high risk of hepatocellular carcinoma. JAMA On Call 3:456-463

11. Nasu K, Kuroki Y, Tsukamoto T, Nakajima H, Mori K, Minami M (2012) Diffusion-weighted imaging of surgically resected hepatocellular carcinoma: imaging characteristics and relationship among signal intensity, apparent diffusion coefficient, and histopathologic grade. AJR Am J Roentgenol 193: 438-444

12. Low RN, Gurney J (2012) Diffusion-weighted MRI (DWI) in the oncology patient: value of breathhold DWI compared to unenhanced and gadolinium-enhanced MRI. J Magn Reson Imaging 25:848-858

13. Shingaki N, Tamai H, Mori Y et al (2013) Serological and histological indices of hepatocellular carcinoma and tumor volume doubling time. Mol Clin Oncol 1:977-981

14. Zhao XT, Li WX, Chai WM, Chen KM (2014) Detection of small hepatocellular carcinoma using gadoxetic acid-enhanced MRI: Is the addition of diffusion weighted MRI at 3.0 T beneficial? J Dig Dis 15:137-145 19

15. Park MS, Kim S, Patel J et al (2012) Hepatocellular carcinoma: detection with diffusion-weighted versus contrast-enhanced magnetic resonance imaging in pretransplant patients. Hepatology 56:140-148

\section{Publisher's Note}

Springer Nature remains neutral with regard to jurisdictional claims in published maps and institutional affiliations.

\section{Submit your manuscript to a SpringerOpen ${ }^{\circ}$ journal and benefit from:}

- Convenient online submission

- Rigorous peer review

- Open access: articles freely available online

- High visibility within the field

- Retaining the copyright to your article

Submit your next manuscript at $\boldsymbol{\nabla}$ springeropen.com 